\title{
Intensifying Brillouin distributed fibre sensors using image processing
}

\author{
Marcelo A. Soto*, Jaime A. Ramírez, Luc Thévenaz \\ EPFL Swiss Federal Institute of Technology, Institute of Electrical Engineering \\ SCI STI LT, Station 11, CH-1015 Lausanne, Switzerland \\ *E-mail: marcelo.soto@epfl.ch
}

\begin{abstract}
Image processing is proposed to enhance the performance of Brillouin distributed fibre sensors. The technique exploits the two-dimensional nature of the measurements, so that each frequency-position pair is assimilated to a pixel of a noisy image. Based on the level of redundancy existing in the two-dimensional information, the method offers unmatched denoising capabilities when compared to classic unidimensional denoising methods, even if those ones are consecutively used in distance and frequency domains. With no modification of the basic configuration, up to $\sim 14 \mathrm{~dB}$ SNR improvement is experimentally demonstrated with unobservable loss of spatial resolution. A figure-of-merit of 115'000 is verified.
\end{abstract}

Keywords: Optical fibre sensor, stimulated Brillouin scattering, distributed fibre sensor, image processing

\section{INTRODUCTION}

One of the main features of Brillouin distributed fibre sensors is their capability to measure temperature and strain profiles along very long sensing ranges using metric spatial resolution. Over the past two decades there have been intense research activities to enhance the performance of this kind of sensors ${ }^{1}$. The signal-to-noise ratio (SNR) of Brillouin optical timedomain analysers (BOTDA) has been substantially improved using advanced techniques, such as distributed Raman amplification $^{2}$, optical pulse coding ${ }^{3,4}$ or other kinds of signal processing ${ }^{5}$, especially when those methods are combined in a single system ${ }^{6}$. Among the different methods proposed in the literature, signal processing techniques, such as optical pulse coding ${ }^{3,4}$ and wavelets ${ }^{5}$, have demonstrated to be very efficient tools to remove noise from a unidimensional array of data; and therefore, their use in BOTDA sensing requires processing time-domain traces at each scanned pump-probe frequency offset independently from each other. A 3D map of the Brillouin gain spectrum (BGS) versus distance can thus be obtained with an improved SNR after processing each time-domain trace $e^{3-5}$. Although methods such as time-frequency codes $^{4}$ take advantage of the double scanning (fibre position and pump-probe frequency detuning) required in a BOTDA sensor, the SNR enhancement provided by that method is basically given by the capability of the code to reduce noise in a unidimensional array of data. So far, no two-dimensional signal processing has been applied to Brillouin sensing to take full advantage of the bi-dimensional nature of the data acquisition process. It can therefore be intuitively expected that the use of a two-dimensional processing technique for denoising the data acquired in a BOTDA sensor can offer a higher SNR enhancement compared to traditional (unidimensional) processing methods reported in the literature.

Following this approach for the first time to our knowledge, image processing is here proposed to remove noise from measurements in Brillouin distributed fibre sensors. Data points, measured at each frequency-position pair, are assimilated to pixels of an image corrupted by noise, which is then removed using well-proven $2 \mathrm{D}$ image processing methods. An SNR enhancement up to $\sim 14 \mathrm{~dB}$ is demonstrated using experimental data acquired by a basic BOTDA sensor and applying four among the best-known algorithms for image noise reduction. This way, Brillouin gain profiles over a $100 \mathrm{~km}$ range are obtained in a $200 \mathrm{~km}$ fibre-loop, showing a frequency uncertainty of $1.5 \mathrm{MHz}$ when using $2 \mathrm{k}$ averages and $2 \mathrm{~m}$ spatial resolution. Although the effectiveness of the technique is experimentally verified using a BOTDA sensor, the method can also be used to reduce noise in Brillouin optical time-domain reflectometers (BOTDR) or phase-sensitive OTDRs.

\section{THEORY AND OPERATING PRINCIPLE}

The measurement process in BOTDA sensors requires the acquisition of time-domain traces of the Brillouin gain at different pump-probe frequency offsets $\Delta f$. The process results in a 2D matrix $M(\Delta f, z)$ of data containing the local BGS at each fibre location $z$. The principle of the technique proposed in this paper is to consider the matrix $M$ as an image, which can be represented by a function $f(x, y)$, where $x$ and $y$ are the spatial coordinates on the obtained image. In a general context, the values of an image $f(x, y)$ belong to a unidimensional space for a grayscale image, and to a threedimensional space $(a, b, c)$ for a colour image, where $a, b$ and $c$ depend on the choice of the colour space ${ }^{7}$ (such as RGB, 
HSV, CIE Lab). For distributed sensing the elements of the matrix $M$ are represented by scalar numbers like in a grayscale image, i.e. $M$ contains unidimensional values mapping the measured local Brillouin gain at a given offset $\Delta f$.

Within the field of computer vision ${ }^{7}$, image processing 7,8 is a tool that focuses on methods and algorithms to obtain visually relevant information from an image. This includes methods for image editing and manipulation, enhancement, feature detection and compression, among others ${ }^{7}$. One of the main challenges of image processing is to enhance the features that are important in an image, and dismiss other elements that are of no relevance, such as background objects, noise, shadows or glare. Among several techniques, noise reduction, also called denoising ${ }^{8,9}$, is one of the most important methods, whose objective is to remove background noise from an image, while preserving the detail information. This exactly matches the requirements for improving the data robustness in a Brillouin distributed sensor while preserving all resolutions.

Denoising algorithms are usually based on the definition of sliding neighbourhoods or $2 D$ windows ${ }^{7}$, corresponding to the set of all pixels at $(x, y)$ that are surrounding a certain position $\left(x^{\prime}, y^{\prime}\right)$. The most common windows are square and centred on the position $\left(x^{\prime}, y^{\prime}\right)$ for which the processing is performed. The larger the window, the greater the reduction of image details. The most basic method of noise removal is known as Gaussian Filtering ${ }^{7,8}$ (GF), which is based on replacing the value of $f\left(x^{\prime}, y^{\prime}\right)$ at the centre of a window by a weighted average of $f(x, y)$ inside the window, where the weights are given by a two-dimensional Gaussian function centred at $\left(x^{\prime}, y^{\prime}\right)$. Gaussian filters are 2D linear filters, and therefore, any increase in the width of the Gaussian function could lead to the unwanted removal of image details. A more sophisticated version of weighted averages is known as Non Local Means ${ }^{8}$ (NLM) algorithm. Similarly to GF, the result of NLM is obtained by weighting the values inside a window centred at $\left(x^{\prime}, y^{\prime}\right)$; however, the weighting factor of a pixel at $(x, y)$ in this case is calculated as the exponential of the Euclidean distance between defined small neighbourhoods around $\left(x^{\prime}, y^{\prime}\right)$ and $(x, y)$, using an exponential decaying factor that has to be properly adjusted ${ }^{8}$. The NLM method can be considered as an improvement respect to Gaussian filters, especially regarding the preservation of edges, texture and fine structures ${ }^{8}$.

Another approach for image denoising uses the frequency domain to separate the components of an image associated with high-frequency noise from the components containing relevant information. Within this category, the 2D Discrete Cosine Transform ${ }^{7,8}$ (DCT) converts the values of each sliding window to the frequency domain, then discards the components that are smaller than a certain threshold level and finally converts the result back to the spatial domain. Another powerful algorithm for image denoising is the 2D Discrete Wavelets Transform ${ }^{8,9}$ (DWT). This method decomposes an image into sub-versions containing different levels of detail and applies to each of them a certain threshold method to eliminate noise. Several parameters, such as the wavelet basis function, the threshold level, and the number of decomposition levels, must be adjusted in a 2D DWT ${ }^{9}$; and hence, all of them have a direct impact on the efficiency of the noise removal.

In this study the four above-mentioned image processing methods are suitably adapted for floating point values to denoise measurements of a BOTDA sensor. The effectiveness of the technique is evaluated by comparing the SNR of the denoised data, while the impact on the spatial resolution is estimated analysing a hot-spot at the far end of the sensing fibre.

\section{EXPERIMENTAL DEMONSTRATION}

As a proof-of-concept experiment, first a basic BOTDA scheme ${ }^{1}$ has been implemented over $50 \mathrm{~km}$ range and $2 \mathrm{~m}$ spatial resolution. A 3D map of the BGS along the fibre has been obtained using 64 time-averaged traces, as shown in Fig. 1(a). It is possible to see that the measured spectrum is highly affected by noise under such a reduced averaging, having a severe impact on the sensor performance ${ }^{1}$. Fig. 1(b) shows a top-view of the measured BGS versus distance. This corresponds to the 'image' to be denoised by image processing. The parameters required by each method have been adjusted aiming at removing only uncorrelated noise, and to avoid any distortion of the BGS and any detrimental impact on the spatial
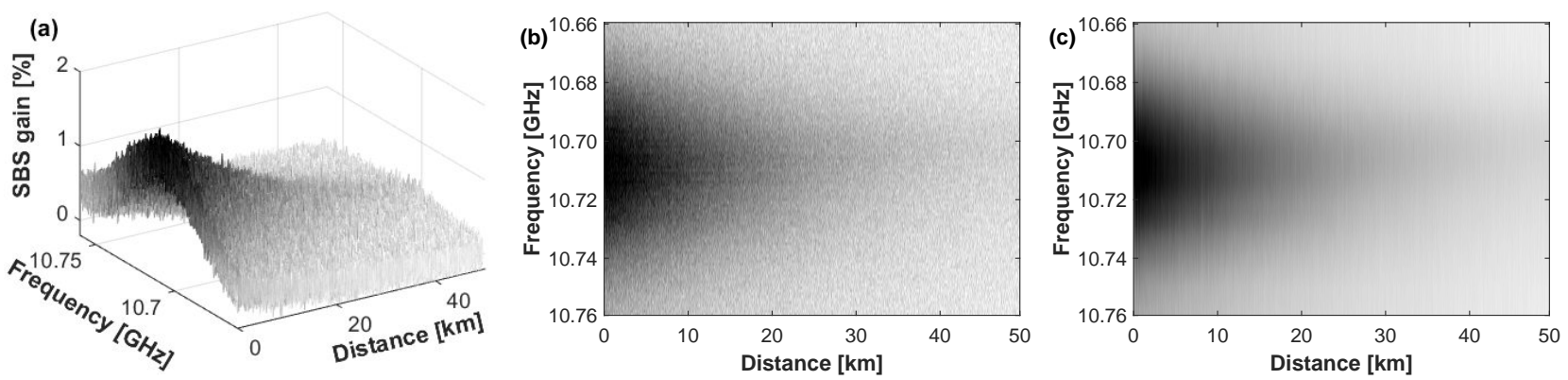

Figure 1. (a) Measured 3D map of the BGS vs distance. (b) Top-view image of the measured BGS vs distance. This can be considered as a noisy image of the ideal BGS. (c) Denoised top-view image of the BGS vs distance using DCT method. 
resolution. After applying GF, NLM, DCT and DWT independently, a significant noise reduction is verified for each case. The effect of the two-dimensional denoising can be seen in Fig. 1(c), which shows the 'denoised image' obtained using the DCT method. The other methods provide similar 'denoised images', also exhibiting large noise reduction. Those images are not shown here for convenience since no visual differences can be perceived when compared to Fig. 1(c).
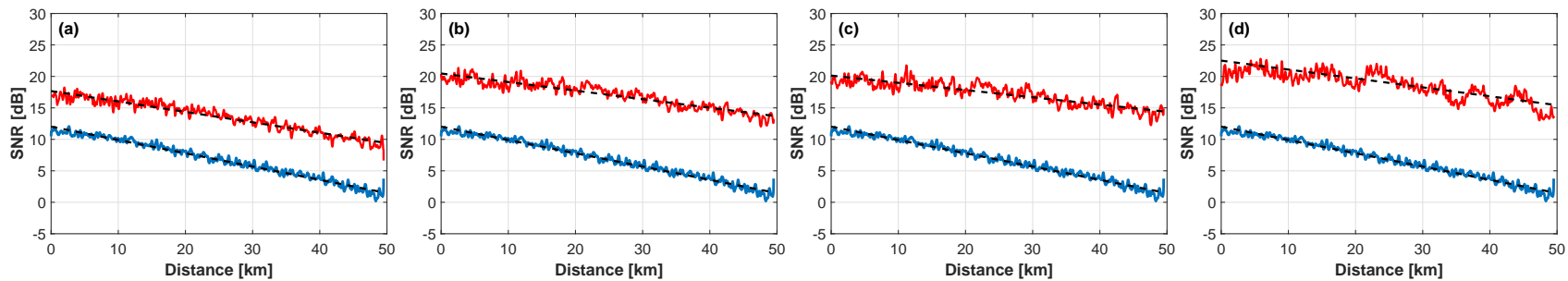

Figure 2. Experimental SNR enhancement provided by the proposed image processing technique. The SNR vs distance (at the peak gain frequency) calculated from the original data (blue curves) is compared with the SNR obtained after image denoising (red curves), for (a) GF, (b) NLM, (c) DCT and (d) DWT methods.

To properly quantify the impact of image processing ${ }^{7-9}$, the SNR of the trace at the peak Brillouin gain has been calculated for each case. Fig. 2 compares the SNR (signal amplitude over standard deviation ${ }^{1}$ ) of the raw data (blue lines) to that obtained after processing (red lines) for the four methods. Results indicate that a raw data SNR of $1.57 \mathrm{~dB}$ obtained at $50 \mathrm{~km}$ distance can be significantly increased up to $9.49 \mathrm{~dB}, 13.73 \mathrm{~dB}, 14.43 \mathrm{~dB}$, and $15.50 \mathrm{~dB}$ using GF, NLM, DCT, and DWT, respectively. Those values represent a remarkable SNR enhancement of $7.92 \mathrm{~dB}, 12.16 \mathrm{~dB}, 12.86 \mathrm{~dB}$, and $13.93 \mathrm{~dB}$ in each of the respective cases. It is worth mentioning that the noise reduction is not always uniform along the fibre, especially for the most efficient methods, such as DCT and DWT. The reason is due to residual polarisation fading affecting the first $\mathrm{km}$ of fibre, which is seen as a correlated noise that is not efficiently eliminated by the image processing. Fortunately this effect only dominates in the first $\mathrm{km}$ of fibre (since polarisation fading is proportional to the Brillouin gain) where the SNR is still high; and hence there is no real detrimental effect at long distances (low SNR region) where the benefit from image denoising is more crucial.

Fig. 3(a) compares the measured BGS near the fibre far end $(50 \mathrm{~km})$ obtained from the raw data (blue curve) and after processing with the DCT method (red curve). It can be observed that the use of image processing leads to a considerable noise reduction with no distortion of the measured BGS. Fig. 3(b) compares the Brillouin frequency shift (BFS) retrieved along the fibre from both raw (blue curve) and denoised data using DCT (red curve). The consequent impact on the sensor performance ${ }^{1}$ has been evaluated calculating the standard deviation of the BFS at each fibre location. Fig. 4 highlights that the frequency uncertainty attained with the raw data (4.7 MHz at $50 \mathrm{~km}$ distance) can be remarkably improved down to $0.77 \mathrm{MHz}$, $0.29 \mathrm{MHz}, 0.25 \mathrm{MHz}$, and $0.20 \mathrm{MHz}$, for the GF, NLM, DCT and DWT methods, respectively. These values are actually in good agreement with the expected uncertainty ${ }^{1}$ based on the experimental SNR reported in Fig. 2.

In order to validate the real benefits of the proposed technique it is essential to verify that these two-dimensional processing methods do not excessively "blur" the 'denoised images', which may result in a loss of spatial resolution for the BOTDA system. To demonstrate this, a 2 m-long hot-spot (placed at the far end of the fibre) is measured with 64 time-averaging and then denoised by the four image processing methods. The BFS profiles obtained after processing are compared with a reference
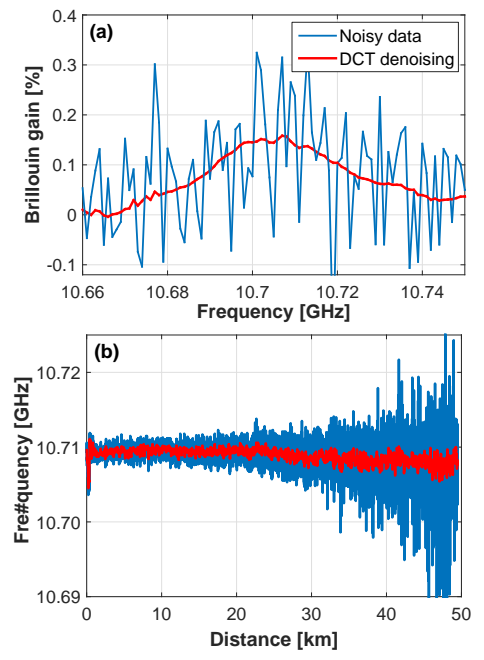

Figure 3. Effect of DCT method on (a) BGS at $50 \mathrm{~km}$ and (b) BFS vs distance. Comparison of raw (blue curves) and denoised data (red curves).
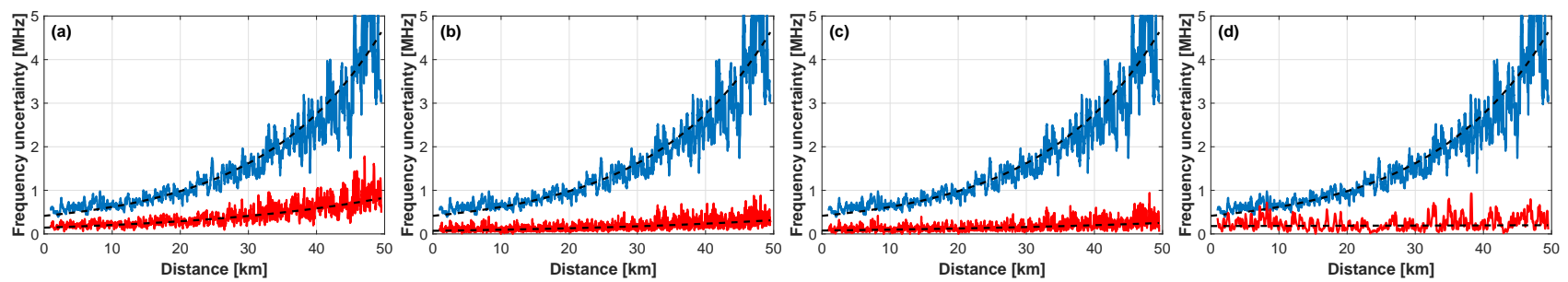

Figure 4. Frequency uncertainty versus distance, calculated as the standard deviation of the retrieved BFS from the original (blue curves) and denoised (red curves) data, for (a) GF, (b) NLM, (c) DCT and (d) DWT methods. 
curve obtained using an advanced BOTDA scheme based on time-frequency multiplexing ${ }^{10}$ and averaging $8 \mathrm{k}$ traces, thus offering a sharp clean reference trace with comparable SNR. Fig. 5 shows the BFS profiles obtained from the sharp reference trace (dashed black curves), the raw measurements (blue curves) and the BFS obtained after image denoising (red curves) for the (a) GF, (b) NLM, (c) DCT and (d) DWT methods. Results show that the hot-spot can be accurately detected in all cases, having a negligible impact on the spatial resolution.
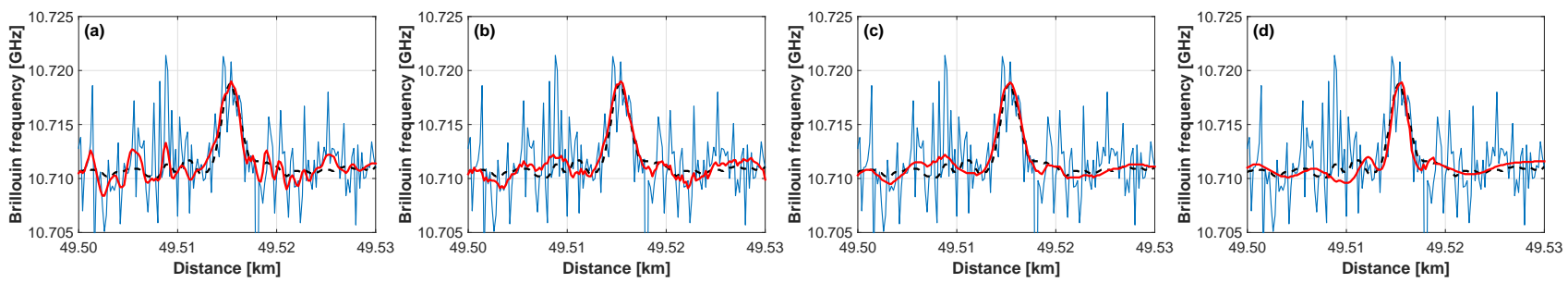

Figure 5. Detection of a $2 \mathrm{~m}$-long hot-spot. Comparison of the BFS obtained from the raw data (blue curve), and the image processing methods (red curve), for (a) GF, (b) NLM, (c) DCT and (d) DWT denoising algorithms. The black dashed line corresponds to a reference curve obtained using an advanced BOTDA scheme and a large number (8k) of averaged traces.

Finally, considering the large SNR enhancement obtained by image denoising, a similar basic BOTDA scheme has been used to cover a sensing range of $100 \mathrm{~km}$ using a $200 \mathrm{~km}$-long fibre-loop (probe experiencing a $42 \mathrm{~dB}$ loss after propagating along the fibre), keeping a spatial resolution of $2 \mathrm{~m}$. Fig. 6 shows a $2 \mathrm{~m}$ hot-spot measured at the end of the sensing fibre after denoising with the NLM method. With no feature added to the standard scheme, the method provides a frequency uncertainty of $1.5 \mathrm{MHz}$ at $100 \mathrm{~km}$ distance when using $2 \mathrm{k}$ averages and $1 \mathrm{MHz}$ scanning step. This corresponds to a figure-of-merit ${ }^{1}$ (FoM) of $115^{\prime} 000$, which is the highest FoM reached using no hardware sophistication in BOTDA sensing.

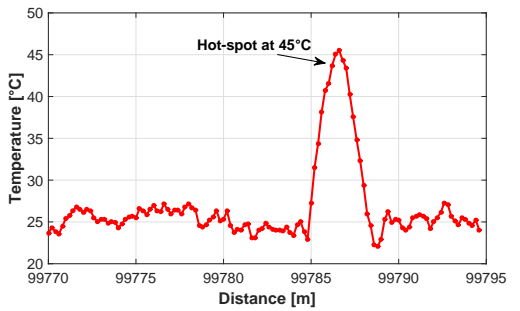

Figure $6.2 \mathrm{~m}$ hot-spot detection at $100 \mathrm{~km}$ distance using a $200 \mathrm{~km}$-long fibre loop, In conclusion, a novel technique, based on the use of image processing, has been $2 \mathrm{k}$ averages, $2 \mathrm{~m}$ spatial resolution and proposed and experimentally validated to reduce noise and very substantially NLM method for signal denoising. improve the performance of Brillouin distributed fibre sensors. A remarkable SNR enhancement (up to about $14 \mathrm{~dB}$ ) has been demonstrated using a simple conventional BOTDA scheme, allowing for measurements over a $100 \mathrm{~km}$ sensing range using a $200 \mathrm{~km}$ fibre-loop, with no need of special hardware and/or complex configurations. The technique can be readily implemented on any existing Brillouin distributed fibre sensors as a simple added software processing layer, bringing a boost in performance equivalent to extensively complex hardware sophistications, at a fraction of the cost.

\section{REFERENCES}

[1] Soto, M. A. and Thévenaz, L., "Modeling and evaluating the performance of Brillouin distributed optical fiber sensors," Opt. Express 21(25), 31347-31366 (2013).

[2] Angulo-Vinuesa, X., et al., "Raman-Assisted Brillouin Distributed Temperature Sensor over $100 \mathrm{~km}$ featuring 2 meter Resolution and $1.2^{\circ} \mathrm{C}$ Uncertainty," J. Lightwave Technol. 30(8), 1060-1065 (2012).

[3] Soto, M. A., Bolognini, G., Di Pasquale, F., and Thévenaz, L. , "Simplex-coded BOTDA fiber sensor with $1 \mathrm{~m}$ spatial resolution over a $50 \mathrm{~km}$ range," Opt. Lett. 35(2), 259-261 (2010).

[4] Le Floch, S., Sauser, F., Soto, M. A., and Thévenaz, L., "Time/frequency coding for Brillouin distributed sensors," Proc. SPIE 8421, OFS2012 22nd International Conference on Optical Fiber Sensors, 84211J (2012).

[5] Farahani, M., Wylie, M., Castillo-Guerra, E., and Colpitts, B., "Reduction in the Number of Averages Required in BOTDA Sensors Using Wavelet Denoising Techniques," J. Lightwave Technol. 30(8), 1134-1142 (2012).

[6] Soto, M. A., et al., "Extending the Real Remoteness of Long-Range Brillouin Optical Time-Domain Fiber Analyzers," J. Lightwave Technol. 32(1), 152-162 (2014).

[7] Szeliski, R., "Computer Vision: Algorithms and Applications". Springer Science \& Business Media, 2010.

[8] Buades, A., Coll, B., Morel, J. M., "A review of image denoising methods, with a new one," Multiscale Model. Simul. 4(2), 490-530 (2005).

[9] Mallat, S.G., "A theory for multiresolution signal decomposition: the wavelet representation," Pattern Analysis and Machine Intelligence, IEEE Transactions on, 11(7), 674-693 (1989).

[10] Soto, M. A., et al., "Time and frequency pump-probe multiplexing to enhance the signal response of Brillouin optical time-domain analyzers," Opt. Express 22(23), 28584-28595 (2014). 